\title{
Keeping the heart empty and beating improves preservation of hypertrophied hearts for valve surgery
}

\author{
Jian Wang, MD, ${ }^{a, b}$ Hongyu Liu, MD, PhD, ${ }^{b}$ Bo Xiang, DDS, ${ }^{a}$ Gang Li, MD, PhD, a,b Marco Gruwel, PhD, ${ }^{a}$ \\ Michael Jackson, PhD, ${ }^{a}$ Boguslaw Tomanek, PhD, ${ }^{a}$ Tomas A. Salerno, MD, ${ }^{\mathrm{C}}$ Roxanne Deslauriers, $\mathrm{PhD},{ }^{a}$ and \\ Ganghong Tian, MD, PhD
}

Objective: This study was designed to determine whether keeping the heart empty and beating improved myocardial fluid homeostasis and energy metabolism of hypertrophied pig hearts in comparison with cardioplegic arrest.

Methods: Twenty piglets underwent a 12-week ascending aortic banding to induce left ventricular hypertrophy. Isolated hypertrophied hearts were divided into 4 groups ( $\mathrm{n}=5$ in each group). Two groups underwent normothermic normokalemic simultaneous perfusion. The other 2 groups were subjected to normothermic hyperkalemic simultaneous perfusion and used as controls. Intramyocardial hydrostatic pressure was monitored with a microtip pressure transducer. Volumes of intracellular and extracellular compartments and myocardial energy metabolism were monitored by using phosphorus 31 magnetic resonance spectroscopy.

From the Institute for Biodiagnostics, ${ }^{\mathrm{a}} \mathrm{Na}-$ tional Research Council, Winnipeg, Manitoba, Canada; Department of Cardiothoracic Surgery, ${ }^{\mathrm{b}}$ the Fourth Affiliated Hospital, Harbin Medical University, Harbin, People's Republic of China; and Division of Cardiothoracic Surgery, ${ }^{\mathrm{C}}$ University of Miami, Miller School of Medicine, and Jackson Memorial Hospital, Miami, Fla.

This work was supported by the Canadian Institutes of Health Research and the National Research Council Canada.

Received for publication March 22, 2006; revisions received July 6, 2006; accepted for publication July 13, 2006.

Address for reprints: Ganghong Tian, MD, $\mathrm{PhD}$, Institute for Biodiagnostics, $435 \mathrm{El}-$ lice Ave, Winnipeg, MB R3B 1Y6, Canada (E-mail: Hong.Tian@nrc-cnrc.gc.ca).

J Thorac Cardiovasc Surg 2006;132:1314-20 $0022-5223 / \$ 32.00$

Copyright (๑) 2006 by The American Association for Thoracic Surgery

doi:10.1016/j.jtcvs.2006.07.010
Results: Normothermic normokalemic simultaneous perfusion (NNSP) maintained intramyocardial hydrostatic pressure at a significantly lower level $(13.0 \pm 0.6 \mathrm{~mm}$ $\mathrm{Hg}$ ) compared with normothermic hyperkalemic simultaneous perfusion (NHSP) $(23.3 \pm 1.2 \mathrm{~mm} \mathrm{Hg})$ during a 90-minute preservation. NNSP maintained the normal volume of the intracellular compartment throughout the preservation period, whereas NHSP caused significant enlargement (to $123 \% \pm 6 \%$ of its normal volume) of the intracellular compartment. Expansion of the extracellular compartment during preservation was significantly less in the NNSP group $(124 \% \pm 6 \%)$ than in the NHSP group $(152 \% \pm 7 \%)$. NNSP maintained normal levels of phosphocreatine and adenosine triphosphate until coronary perfusion flow was reduced to $50 \%$ of the initial control level. No decrease in energy metabolites was observed in the NHSP group even when coronary perfusion flow was reduced to $10 \%$ of the initial control level.

Conclusions: Keeping the heart empty and beating improves myocardial fluid homeostasis for hypertrophied hearts relative to cardioplegic arrest. Its ability to maintain energy metabolism depends on the degree of coronary stenosis. This technique may be a promising protective strategy for hypertrophied hearts.

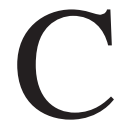
ardiac surgery is still the most effective therapeutic treatment for valve diseases. ${ }^{1}$ In patients with preserved ventricular function, cardioplegic techniques provide sufficient myocardial protection and lead to a complete postoperative recovery of cardiac function. ${ }^{1,2}$ In patients with compromised cardiac function, such as high-risk patients with severe myocardial hypertrophy, cardioplegia may not be able to provide adequate myocardial protection. ${ }^{3,4}$ This is because the hypertrophied heart has less tolerance to the detrimental effects of cardioplegia, such as myocardial edema and overload of potassium and chloride.

Myocardial hypertrophy is associated with a significant enlargement of the myocardial interstitial compartment and a decrease in the density of the capillaries 


$$
\begin{aligned}
& \text { Abbreviations and Acronyms } \\
& \begin{aligned}
\mathrm{ATP} & =\text { adenosine triphosphate } \\
\mathrm{DMMP} & =\text { dimethyl methylphosphonate } \\
\mathrm{LAD} & =\text { left anterior descending coronary artery } \\
\mathrm{LV} & =\text { left ventricle } \\
\mathrm{MR} & =\text { magnetic resonance } \\
\mathrm{NHSP} & =\text { normothermic hyperkalemic simultaneous } \\
& \text { perfusion } \\
\mathrm{NNSP} & =\text { normothermic normokalemic simultaneous } \\
& \text { perfusion } \\
\mathrm{PCr} & =\text { phosphocreatine } \\
\mathrm{PPA} & =\text { phenylphosphonic acid }
\end{aligned}
\end{aligned}
$$

and mitochondria. ${ }^{5-8}$ These pathologic changes provide the histologic foundation for severe myocardial edema and compromised myocardial perfusion and energy metabolism. Moreover, cardioplegia abolishes the squeezing effect of myocardial contraction on the coronary system, which is an important element in maintenance of normal myocardial fluid homeostasis and blood perfusion. ${ }^{9,10}$ Cardioplegia results in an increase in concentrations of potassium and chloride and also causes water accumulation in both the extracellular and intracellular compartments. ${ }^{11,12}$ Thus, cardioplegic techniques may offer only suboptimal protection for hypertrophied hearts in high-risk patients.

The development of beating coronary artery bypass grafting has indicated that cardioplegia is not an indispensable component for cardiac surgery. By extension, it is believed that valve surgery can also be performed under beating conditions. Masroor and colleagues ${ }^{13}$ and Di Luozzo and associates ${ }^{14}$ have successfully adopted this technique in their clinical practice. Their preliminary clinical experience has suggested that keeping the heart empty and beating during valve surgery is advantageous in high-risk patients. ${ }^{13,14}$ We hypothesized that the potential beneficial effects of keeping the heart empty and beating are closely related to improved preservation of myocardial fluid homeostasis and the volume of the cellular compartments. This study was therefore designed to test our hypothesis.

Partington and associates ${ }^{15,16}$ elegantly showed that ideal myocardial protection requires the administration of cold blood cardioplegia alternating between antegrade and retrograde infusions, because each of these modalities of cardioplegic administration perfuses different vessel beds of the myocardium. Subsequently, Ihnken and associates ${ }^{17,18}$ introduced the concept of simultaneous antegrade/retrograde warm blood cardioplegia. They concluded that this was a "near" ideal method of myocardial protection, a technique that was investigated both in animals and humans. Thus, simultaneous perfusion was chosen as a perfusion technique for this study.

\section{Materials and Methods}

The animals used in this study received humane care in compliance with the Guide to the Care and Use of Experimental Animals formulated by the Canadian Council on Animal Care. The Animal Care Committee in the National Research Council approved the experimental protocols used in this study.

\section{Pig Model of Pressure-overloaded Left Ventricular Hypertrophy}

Twenty 6- to 8-week-old piglets weighing 5 to $7 \mathrm{~kg}$ were sedated with an intramuscular injection of diazepam $(0.4 \mathrm{mg} / \mathrm{kg}$ body weight $)$ and ketamine ( $20 \mathrm{mg} / \mathrm{kg}$ body weight). After the induction of anesthesia, piglets were intubated and mechanically ventilated with gas anesthesia comprising $1 \%$ to $2 \%$ isoflurane in a mixture of oxygen and nitrous oxide. A left lateral thoracotomy was performed in the third intercostal space. The pericardium was incised, and care was taken to avoid damage to the phrenic nerve. The ascending aorta was dissected bluntly from pulmonary artery. A piece of suture inside a silicone tube was placed to circle the ascending aorta. The ends of the suture were tied to allow for overlap of the tube ends to create a peak systolic pressure gradient of 10 to $20 \mathrm{~mm} \mathrm{Hg}$ between the left ventricle (LV) and aorta distal to the stenosis. The chests were closed, and the animals were allowed to recover for 12 weeks for the development of LV hypertrophy.

\section{Isolated Pig Heart Preparation}

Twelve weeks after banding of the aorta, the animals' chests and pericardia were reopened under general anesthesia. The aorta, pulmonary artery, and inferior and superior vena cava were dissected and clamped. A heparinized polyethylene catheter was inserted into the LV chamber to measure intraventricular pressure. Cold $\left(4^{\circ} \mathrm{C}\right)$ cardioplegia was infused into the aortic root to arrest the heart. The heart was quickly excised and immersed in cold saline solution for instrumentation. The aorta and left anterior descending (LAD) coronary artery were cannulated for antegrade perfusion. A $17 \mathrm{~F}$ retrograde cannula was positioned in the coronary sinus and secured with a purse-string suture for retrograde perfusion. A short piece of polyethylene was inserted into the LV through the apex to keep the LV empty throughout the protocol.

Pig blood in the animal chest was collected and mixed with Krebs-Henseleit solution in a 1:1 ratio for perfusion of the pig heart. The Krebs-Henseleit solution contained $118 \mathrm{mmol} / \mathrm{L} \mathrm{NaCl}$, $1.2 \mathrm{mmol} / \mathrm{L} \mathrm{MgSO}_{4}, 0.5 \mathrm{mmol} / \mathrm{L}$ ethylenediaminetetraacetic acid, $11 \mathrm{mmol} / \mathrm{L}$ glucose, $25 \mathrm{mmol} / \mathrm{L} \mathrm{NaHCO}_{3}, 1.75 \mathrm{mmol} / \mathrm{L} \mathrm{CaCl}_{2}$, and $0.625 \%$ bovine serum albumin. The concentration of potassium was 4.0 and $16 \mathrm{mmol} / \mathrm{L}$ for normothermic normokalemic simultaneous perfusion (NNSP) and normothermic hyperkalemic simultaneous perfusion (NHSP), respectively. The mixture was oxygenated with $95 \%$ oxygen and $5 \%$ carbon dioxide to a final $\mathrm{pH}$ and $\mathrm{Po}_{2}$ of $7.42 \pm 0.02 \mathrm{pH}$ units and $604.7 \pm 25 \mathrm{~mm} \mathrm{Hg}$, respectively. The temperature of the heart was maintained at $36.5^{\circ} \mathrm{C}$ to $37^{\circ} \mathrm{C}$ throughout the protocol.

\section{Experimental Protocols}

Isolated hypertrophied pig hearts were divided into 4 groups with 5 hearts in each group. Groups I and II were used to assess the effects of empty beating (NNSP) and cardioplegic arrest (NHSP) on intramyocardial hydrostatic pressure and the volumes of the 
Group I (NNSP) and Group I (NHSP)

\begin{tabular}{|c|c|c|}
\hline Protocol & 10 -minute CP & 90-minute NNSP or NHSP \\
\hline LAD flow & LAD full flow (perfusion pressure of $60 \mathrm{mmHg}$ ) \\
\hline Measurements & $\begin{array}{l}\text { Measure volumes of the extracellul ar and intracellul ar compartments } \\
\text { and intra-myocardial hydrostatic pressure }\end{array}$ \\
\hline
\end{tabular}

Group II (NNSP) and Group IV (NHSP)

\begin{tabular}{|c|c|c|c|c|c|c|c|c|c|}
\hline Protocol & 10 -minute CP & \multicolumn{7}{|c|}{65 -minute NNSP or NHSP } \\
\hline LAD flow & $100 \%$ & $100 \%$ & $70 \%$ & $100 \%$ & $50 \%$ & $100 \%$ & $30 \%$ & $100 \%$ & $10 \%$ \\
\hline Time & $10 \mathrm{~min}$ & $10 \mathrm{~min}$ & $10 \mathrm{~min}$ & $5 \mathrm{~min}$ & $10 \mathrm{~min}$ & $5 \mathrm{~min}$ & $10 \mathrm{~min}$ & $5 \mathrm{~min}$ & $10 \mathrm{~min}$ \\
\hline Measurements & \multicolumn{7}{|c|}{ Monitor myocar dial energy metabolism } \\
\hline
\end{tabular}

Figure 1. Schematic illustration of the experimental protocol. $C P$, Control perfusion; NNSP, normothermic normokalemic simultaneous perfusion; NHSP, normothermic hyperkalemic simultaneous perfusion; LAD, left anterior descending coronary artery.

cellular compartments. The hearts in the 2 groups underwent a protocol consisting of a 10-minute antegrade normokalemic perfusion (control perfusion) and 90 minutes of either NNSP (group I) or NHSP (group II). A microtip pressure transducer with a needle tip was inserted 4 to $5 \mathrm{~mm}$ into the LV wall for measurement of intramyocardial hydrostatic pressure. Perfusion pressure at the aorta and coronary sinus was maintained at 60 and $40 \mathrm{~mm} \mathrm{Hg}$, respectively. The corresponding total antegrade flow was $217 \pm 13$ $\mathrm{mL} / \mathrm{min}$, and retrograde flow was $47 \pm 4 \mathrm{~mL} / \mathrm{min}$ throughout the simultaneous perfusion period.

Dimethyl methylphosphonate (DMMP; $20 \mathrm{mmol} / \mathrm{L}$ ) and phenylphosphonic acid (PPA; $10 \mathrm{mmol} / \mathrm{L}$ ) were added into the mixture (pig blood and Krebs-Henseleit solution) to monitor the volumes of the intracellular and extracellular compartments. Both compounds are chemically inert and nontoxic. DMMP rapidly distributes to the entire water compartment, whereas PPA distributes only into the extracellular compartment. ${ }^{19,20}$ The difference between DMMP and PPA distribution volumes gives rise to the volume of the intracellular compartment. Changes in the volumes of the 2 compartments during NNSP or NHSP were monitored by following the magnetic resonance (MR) signal intensities of the 2 chemicals. ${ }^{21}$ A small plastic ball filled with $1 \mathrm{~mL}$ of $500 \mathrm{mmol} / \mathrm{L}$ methylphosphonic acid was placed in the right ventricles as a reference for quantification of DMMP and PPA.

Groups III and IV were used to assess the ability of empty beating (NNSP) and cardioplegic arrest (NHSP) to maintain myocardial energy metabolism. The LAD was cannulated in the 2 groups of hearts to control blood flow. Hearts in both groups were first subjected to a 10-minute control perfusion and then to 65 minutes of NNSP (group III) or NHSP (group IV). During the simultaneous perfusion, LAD blood flow gradually decreased from $100 \%$ to $70 \%, 50 \%, 30 \%$, and $10 \%$ of the initial control level obtained at a perfusion pressure of $60 \mathrm{~mm} \mathrm{Hg}$. The decrease in LAD perfusion flow was used to simulate coronary stenosis. Per- fusion at each flow rate lasted 10 minutes. A 5-minute perfusion at $100 \%$ flow was used before any decrease in LAD perfusion flow. Again, perfusion pressures at the aorta and coronary sinus were kept at 60 and $40 \mathrm{~mm} \mathrm{Hg}$, respectively, during the 65-minute simultaneous perfusion.

At the end of the experiment, total heart weight, LV weight (LV free wall and septum), and areas of the aortic root and the banding site of the hypertrophied hearts were measured. The parameters of normal hearts (with no aortic banding) were obtained from the animals in our other studies and used as control references. Experimental protocols are illustrated in Figure 1.

\section{Phosphorus 31 MR Spectroscopy}

Phosphorus $31\left({ }^{31} \mathrm{P}\right)$ MR spectroscopy was performed on a 7-T magnet equipped with a Biospec spectrometer (Bruker, Karlsruhe, Germany). A global MR coil surrounding the entire heart was used in groups I and II. Thus, the MR signals were acquired from the entire pig heart. In groups III and IV, an MR surface coil with a $1.5-\mathrm{cm}$ diameter was positioned over the LAD region. The MR signals were therefore acquired only from myocardium supported by the LAD.

MR signals (free induction decay) were obtained by use of a hard pulse with a pulse length of 75 microseconds and a repetition time of 2 seconds. Six free induction decay signals were accumulated for each ${ }^{31} \mathrm{P}$ MR spectrum. Thus, each spectrum was averaged over a 2-minute sample time.

The observed phosphorus compounds included DMMP, PPA, methylphosphonic acid, inorganic phosphate, phosphocreatine (PCr), and 3 peaks ( $\alpha, \beta$, and $\gamma$ ) of adenosine triphosphate (ATP). The $\beta$ peak was used for quantifying ATP.

\section{Data Analysis}

The ${ }^{31} \mathrm{P}$ MR spectra were analyzed by using the software $1 \mathrm{D}$ WINNER (Bruker). Myocardial energy metabolites ( $\mathrm{PCr}$ and 
ATP), DMMP, and PPA are expressed as the percentage of their initial levels obtained during the control perfusion.

Statistical analyses were performed with Statistica (StatSoft Inc, Tulsa, Okla). All numerical data are expressed as the mean \pm SD. One-way analysis of variance was used to compare the changes in the intracellular and extracellular compartments and the intramyocardial hydrostatic pressure between groups I and II. One-way analysis of variance was also used to compare the levels of myocardial PCr and ATP in groups III and IV.

\section{Results}

Myocardial Hypertrophy

The area of the aortic banding site was $0.8 \pm 0.3 \mathrm{~cm}^{2}$, and the area of the aortic root was $2.4 \pm 0.6 \mathrm{~cm}^{2}$, thus indicating an approximately $60 \%$ reduction in aortic cross-sectional area. LV end-diastolic pressure was significantly $(P=$ $.0014)$ higher in the hypertrophied $(15.2 \pm 3.7 \mathrm{~mm} \mathrm{Hg})$ than in the normal $(5.1 \pm 1.3 \mathrm{~mm} \mathrm{Hg})$ hearts. The ratio of $\mathrm{LV}$ weight to entire heart weight was also significantly $(P=$ $.011)$ higher in hypertrophied hearts $(0.57 \pm 0.04)$ than in normal hearts $(0.46 \pm 0.03)$. Heart weight was greater $(P=$ $.0031)$ in hypertrophied hearts $(298 \pm 54 \mathrm{~g})$ than in normal hearts $(236 \pm 40 \mathrm{~g})$. The index of heart weight to body weight was also significantly $(P=0.0032)$ higher in the hypertrophied hearts $(6.91 \pm 0.79)$ than in the normal hearts $(5.97 \pm 0.57)$. These suggest that 12 -week aortic banding resulted in significant LV hypertrophy.

\section{Effect of NNSP and NHSP on Intramyocardial Hydrostatic Pressure}

A representative intramyocardial hydrostatic pressure record obtained from a heart undergoing NNSP and NHSP is shown in the upper panel of Figure 2. Intramyocardial pressure varied with contraction and relaxation of the heart. Measurements at the end of diastole represented intramyocardial hydrostatic pressure during NNSP. It was very clear that cardioplegic arrest achieved with NHSP resulted in rapid and significant elevation of intramyocardial hydrostatic pressure. Averages of intramyocardial hydrostatic pressure measured from the NNSP hearts $(13 \pm 0.6 \mathrm{~mm} \mathrm{Hg})$ were significantly $(P=.0037)$ lower than those obtained from the NHSP hearts $(23 \pm 1.2 \mathrm{~mm} \mathrm{Hg})$ (Figure 2, lower panel).

\section{Effect of NNSP and NHSP on the Volumes of the Intracellular and Extracellular Compartments}

Changes in the volumes of the cellular compartments during 90 minutes of NNSP and NHSP are shown in Figure 3. A 90-minute NHSP resulted in a progressive and significant increase in the volume of both the intracellular and extracellular compartments (Figure 3). At the end of NHSP, volumes of the intracellular and extracellular compartments were $123 \% \pm 6 \%(P=.00091$ vs its initial value of $100 \%)$ and $152 \% \pm 7 \%(P=.00003$ vs its initial value of $100 \%)$, respectively. This suggests that prolonged NHSP is associ-
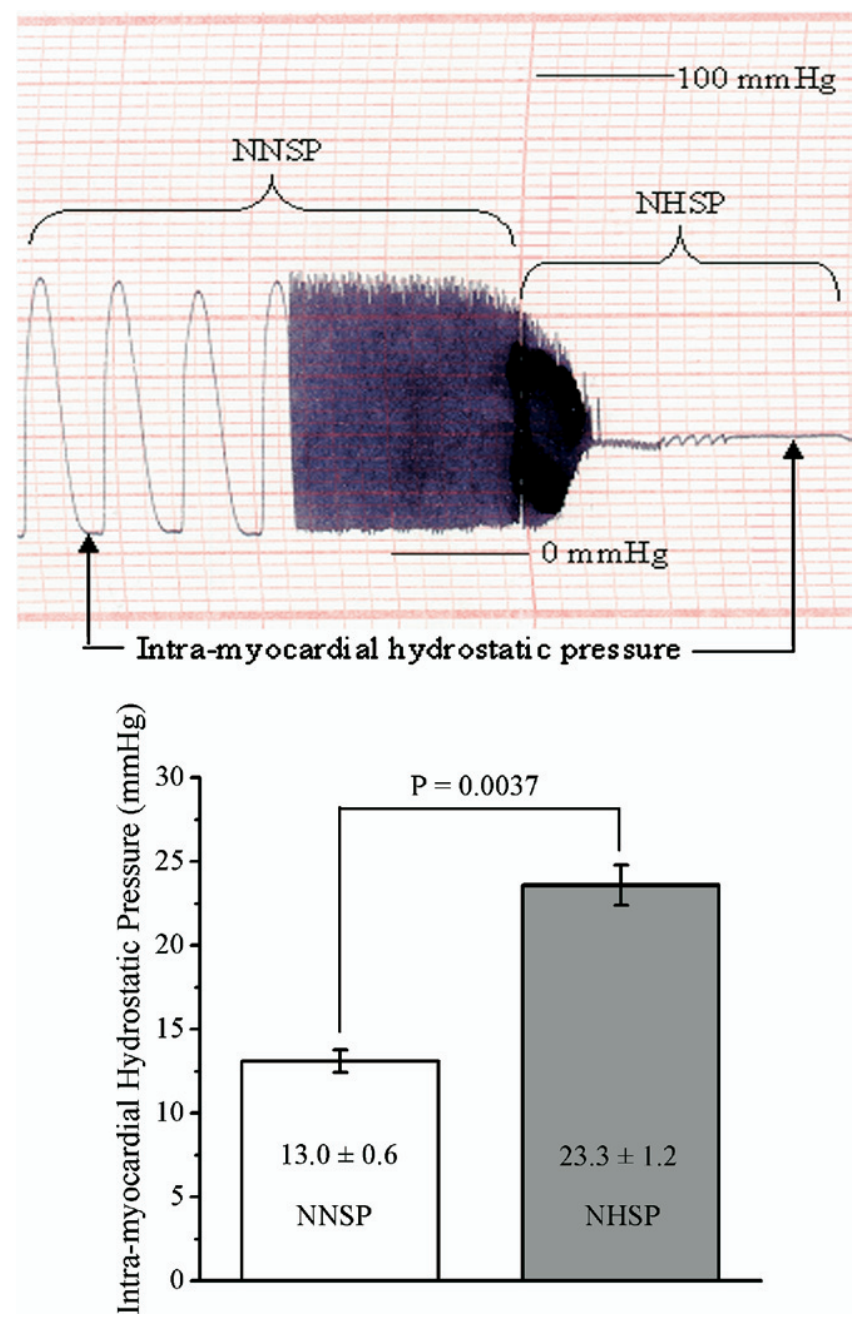

Figure 2. The upper panel shows a representative intramyocardial pressure record obtained from a heart subjected to NNSP and NHSP. The lower panel shows average intramyocardial hydrostatic pressures obtained from NNSP hearts and NHSP hearts. $N N S P$, normothermic normokalemic simultaneous perfusion; NHSP, normothermic hyperkalemic simultaneous perfusion.

ated with significant myocardial edema, with water accumulating in both compartments. In comparison with the NHSP hearts, the NNSP hearts showed significantly $(P=$ $.00041)$ less expansion of the extracellular compartment. At the end of NNSP, the volume of the extracellular compartment was $124 \% \pm 6 \%$ of its initial volume (Figure 3 , upper panel). More importantly, the volume of the intracellular compartment remained relatively unchanged throughout the 90-minute NNSP (Figure 3, lower panel).

Efficacy of NNSP and NHSP for Sustaining Myocardial Energy Metabolism

Representative ${ }^{31} \mathrm{P}$ MR spectra obtained from NNSP and NHSP hearts are shown in Figure 4. Average levels of 

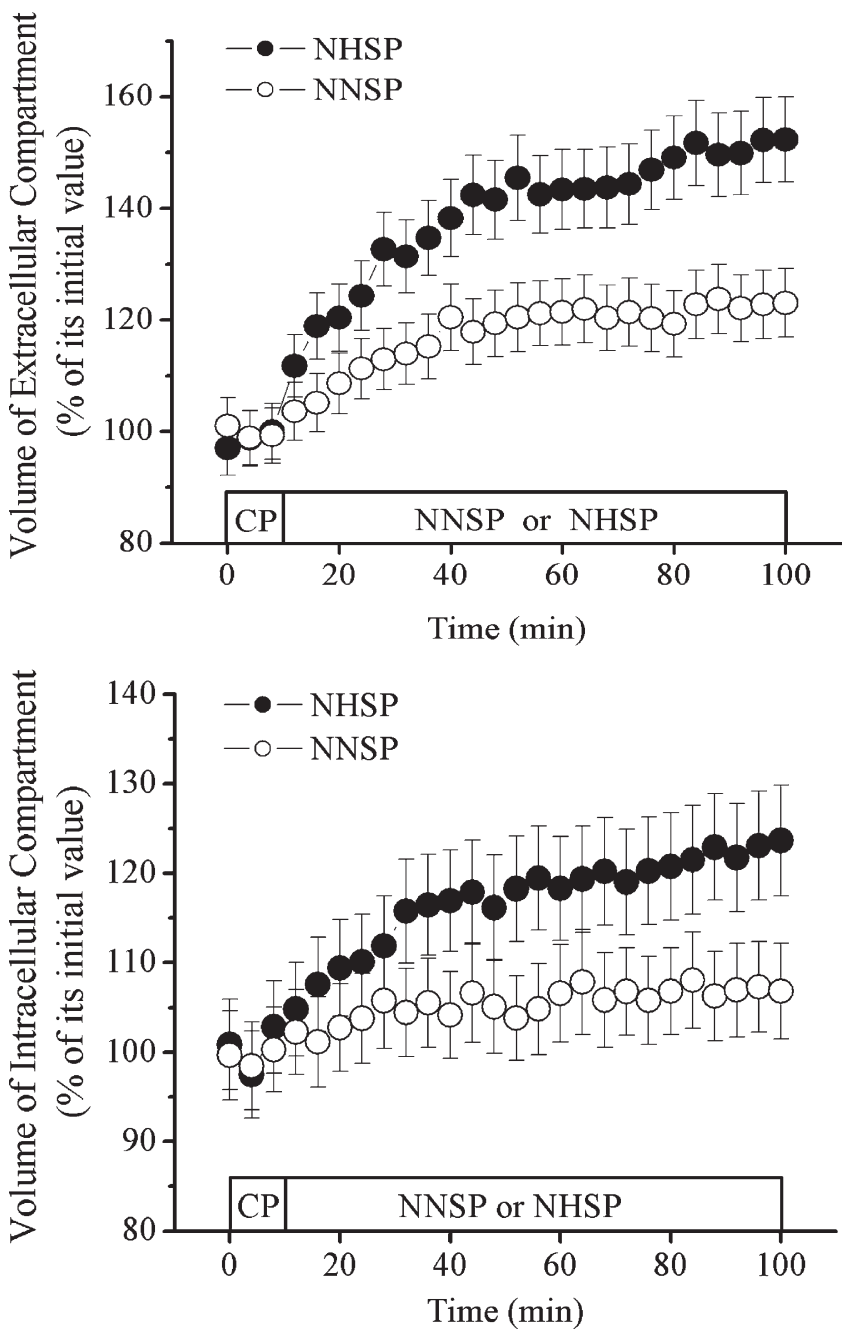

Figure 3. Comparison of the volumes of extracellular (upper panel) and intracellular (lower panel) compartments between the NNSP hearts and NHSP hearts. NHSP, normothermic hyperkalemic simultaneous perfusion; NNSP, normothermic normokalemic simultaneous perfusion; $C P$, Control perfusion.

myocardial PCr and ATP measured at different LAD blood flows in both groups of hearts are shown in Figure 5. With LAD blood flow more than $50 \%$ of the initial control level, NNSP maintained normal levels of myocardial PCr and ATP. Decreased PCr in NNSP hearts became significant when LAD blood flow was decreased to $50 \%$ of the initial control level $(P=.0038$ vs the $\mathrm{PCr}$ level measured at $100 \%$ LAD flow). A significant reduction in the ATP level was observed only when the LAD flow was reduced to $30 \%$ of the initial control level $(P=$ .0023). In contrast, hearts subjected to NHSP did not show any significant decrease in PCr and ATP levels even with LAD blood flow reduced to $10 \%$ of the initial control level.

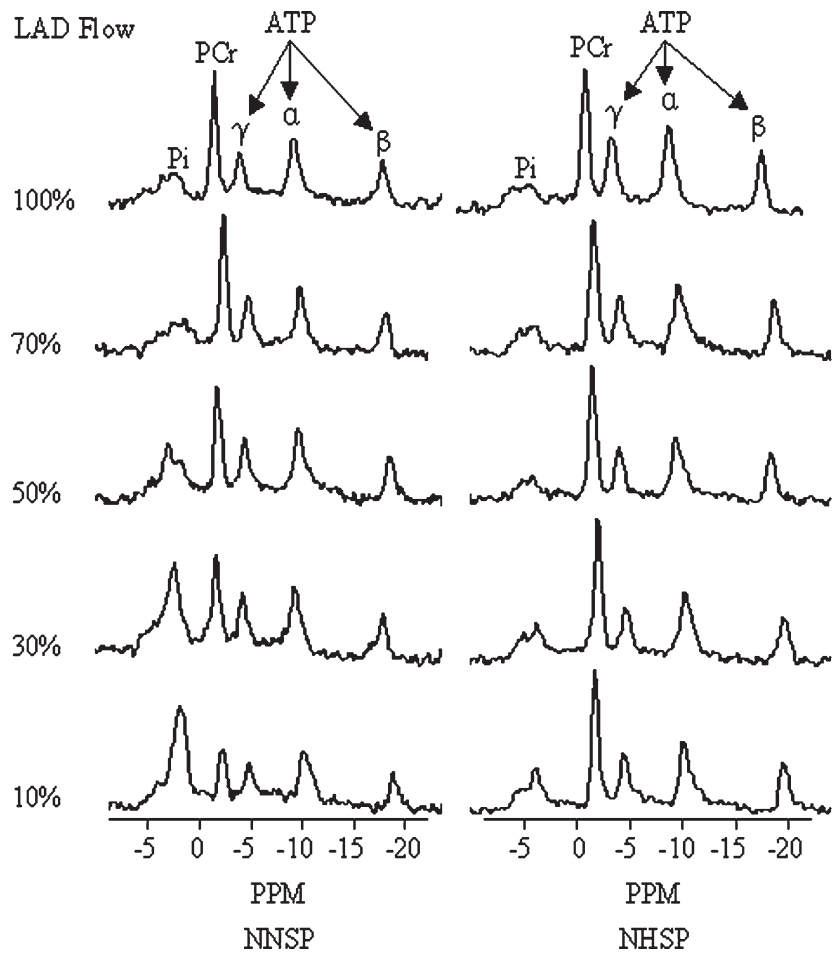

Figure 4. Representative ${ }^{31} \mathrm{P}$ MR spectra obtained from an NNSP heart and an NHSP heart. A significant increase in inorganic phosphate $(P i)$ and a decrease in PCr were observed in the NNSP heart when the LAD flow was reduced to $50 \%$ of its control level (left column). No significant changes in ATP, $\mathrm{PCr}$, or $\mathrm{Pi}$ were observed in the NHSP heart (right column). LAD, left anterior descending coronary artery; $A T P$, adenosine triphosphate; $P P M_{\text {, }}$ parts per million; NNSP, normothermic normokalemic simultaneous perfusion; NHSP, normothermic hyperkalemic simultaneous perfusion.

\section{Discussion}

Cardiac surgery has provided a reliable treatment to correct valve abnormalities and to alleviate the life-threatening symptoms and complications of valve diseases. ${ }^{1}$ At present, myocardial protection during valve surgery relies primarily on various cardioplegic techniques. However, even with continuous warm blood cardioplegia, which is considered to be the best form of myocardial protection, ${ }^{22,23}$ some degree of postoperative myocardial dysfunction may still occur. ${ }^{24}$ This suggests that cardioplegia does not provide optimal myocardial preservation, especially for hypertrophied hearts. ${ }^{3,4}$ The detrimental effects of cardioplegia on tissue fluid homeostasis, volumes of cellular compartments, and myocardial perfusion may be the underlying mechanisms for postoperative cardiac dysfunction. To improve preservation of the hypertrophied heart, keeping heart empty beating has been proposed as an alternative cardioprotective technique. This study was performed to evaluate the effects 

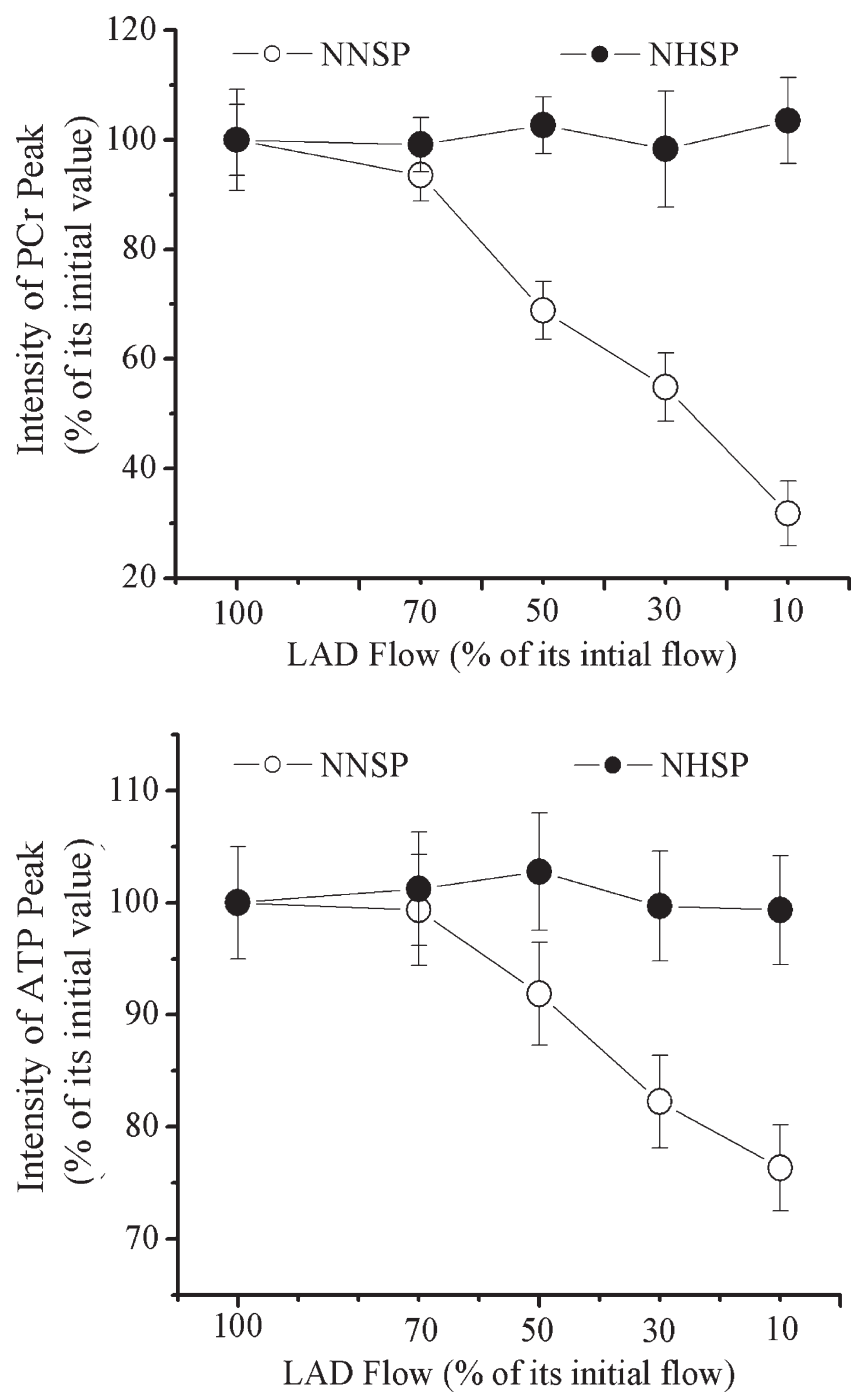

Figure 5. Comparison of myocardial PCr (upper panel) and ATP (lower panel) levels between the NNSP hearts and NHSP hearts with differential LAD perfusion flow. NNSP, normothermic normokalemic simultaneous perfusion; NHSP, normothermic hyperkalemic simultaneous perfusion; $L A D$, left anterior descending coronary artery; $A T P$, adenosine triphosphate.

of this technique on myocardial fluid homeostasis, the volume of the cellular compartments, and energy metabolism.

It has been shown that myocardial hypertrophy is associated with an approximately $30 \%$ enlargement of the interstitial compartment, a $100 \%$ increase in myocardial collagen, and a $50 \%$ increase in proteoglycan. ${ }^{5,6}$ Because interstitial fluid is mainly entrapped in minute spaces among the proteoglycan filaments, enlargement of the interstitial compartment and an increase in collagen and proteoglycan provide a histologic foundation for severe tissue edema. Therefore, cardioplegia-resulting destruction of myocardial fluid homeostasis is expected to be more severe in hypertrophied than in nonhypertrophied hearts.

NNSP prevented an increase in intramyocardial hydrostatic pressure and significantly reduced enlargement of the extracellular compartment. It maintained a normal volume of the intracellular compartment throughout 90 minutes of NNSP. In contrast, NHSP significantly increased intramyocardial hydrostatic pressure and resulted in enlargement of both the intracellular and extracellular compartments. We believe that the increase in intramyocardial hydrostatic pressure and the expansion of both cellular compartments in the NHSP hearts were the result of cardioplegia-induced fluid accumulation. It has been shown that cardiac arrest significantly decreases myocardial lymph flow and impairs lymph drainage, thus leading to water accumulation in the interstitial compartment. ${ }^{9,10,25,26}$ Decreased lymph flow and driving pressure were observed during ventricular fibrillation, while normal coronary perfusion was maintained. ${ }^{10,25}$ This suggests that organized myocardial contraction is very important for maintenance of normal myocardial lymph flow and myocardial fluid homeostasis. ${ }^{10,25}$ In addition, as a result of prolonged diastolic cardiac arrest, myocardial microvascular filtration was increased, thus leading to the development of myocardial edema during cardioplegia. ${ }^{27}$ NNSP maintained normal electromechanical activity and lymph flow of the hearts, thus preventing water accumulation within both extracellular and intracellular compartments. Moreover, according to the Donnan relationship, an increased concentration of potassium, chloride, and water in the interstitial compartment leads to increased movement of the ions and water molecules into the intracellular compartment. ${ }^{11,12}$ NNSP avoided hyperkalemia and hyperchloremia. Thus, NNSP maintained a normal intracellular compartment and minimized myocardial edema.

Cardioplegia abolishes the electromechanical activity of the heart and reduces the myocardial energy demand by a factor of approximately $100 .{ }^{2}$ As such, hearts subjected to NHSP did not show any significant change in the levels of ATP and PCr with the LAD perfusion flow reduced. In contrast, the hearts subjected to NNSP started to show a decline in the PCr level when the LAD perfusion flow decreased to $50 \%$ of the initial control level. Further decreases in the LAD perfusion flow caused a linear reduction in ATP and PCr levels. Thus, NNSP was inferior to cardioplegia (NHSP) in maintaining myocardial energy metabolism; this was anticipated. Nevertheless, NNSP was able to sustain the normal myocardial energy metabolism of hypertrophied hearts even in stimulated moderate coronary stenosis. Because PCr is normally used to replenish ATP during ischemia, a striking decrease in the $\mathrm{PCr}$ level started earlier than a significant decrease in the ATP level. ${ }^{28-30}$

In this study, histologic assessment of the hypertrophied heart was not performed. Thus, no histologic evidence of 
myocardial hypertrophy was provided. However, hemodynamic and heart weight data indicated significant myocardial hypertrophy. In addition, isolated pig hearts were perfused with highly diluted pig blood in a Langendorff apparatus. Therefore, the myocardial edema and expansion of cellular compartments observed in this study may be much more severe than those encountered in hypertrophied human hearts. Nevertheless, our results demonstrate that keeping the heart beating with NNSP improved preservation for hypertrophied hearts in comparison with cardioplegic arrest using NHSP. The effects of keeping the heart empty and beating on myocardial perfusion and contractile function are currently under investigation in our laboratory.

In summary, keeping the heart empty and beating with normothermic normokalemic simultaneous blood perfusion improved the preservation of myocardial fluid homeostasis and the volumes of the cellular compartments in comparison with cardioplegic arrest using normothermic hyperkalemic simultaneous antegrade/retrograde blood perfusion. The first basic study on this new technique of myocardial protection forms the basis for the further investigation needed to fully delineate its potential benefits for surgery on the hypertrophied heart.

The authors thank Allan Turner, Lori Gregorash, and Shelley Germscheid for their assistance with surgery.

\section{References}

1. Letsou GV, Reardon MJ. Minimally invasive valve surgery. Curr Opin Cardiol. 1998;13:105-10.

2. Gebhard MM, Brestchneider HJ, Schnabel PA. Cardioplegia principles and problems. In: Sperelakis N, editor. Physiology and pathophysiology of the heart. 2nd ed. Boston: Kluwer Academic; 1989. p. 655-69.

3. Ascione R, Caputo M, Gomes WJ, Lotto AA, Bryan AJ, Angelini GD, et al. Myocardial injury in hypertrophic hearts of patients undergoing aortic valve surgery using cold or warm blood cardioplegia. Eur J Cardiothorac Surg. 2002;21:440-6.

4. Lotto AA, Ascione R, Caputo M, Bryan AJ, Angelini GD, Suleiman MS. Myocardial protection with intermittent cold blood during aortic valve operation: antegrade versus retrograde delivery. Ann Thorac Surg. 2003;76:1227-33.

5. Rothermund L, Kreutz R, Kossmehl P, Fredersdorf S, Shakibaei M, Schulze-Tanzil G, et al. Early onset of chondroitin sulfate and osteopontin expression in angiotensin II-dependent left ventricular hypertrophy. Am J Hypertens. 2002;15:644-52.

6. Anversa P, Capasso JM. Loss of intermediate-sized coronary arteries and capillary proliferation after left ventricular failure in rats. Am J Physiol. 1991;260:H1552-60.

7. Tomanek RJ, Palmer PJ, Peiffer GL, Schreiber KL, Eastham CL, Marcus ML. Morphometry of canine coronary arteries, arterioles, and capillaries during hypertension and left ventricular hypertrophy. Circ Res. 1986;58:38-46.

8. Kayar SR, Weiss HR. Diffusion distances, total capillary length and mitochondrial volume in pressure-overload myocardial hypertrophy. $J$ Mol Cell Cardiol. 1992;24:1155-66.

9. Ullal SR, Kluge TH, Gerbode F. Studies on cardiac lymph during extracorporeal circulation. Chest. 1972;61:662-7.
10. Mehlhorn U, Davis KL, Burke EJ, Adams D, Laine GA, Allen SJ Impact of cardiopulmonary bypass and cardioplegic arrest on myocardial lymphatic function. Am J Physiol. 1995;268:H178-83.

11. Drewnowska K, Clemo HF, Baumgarten CM. Prevention of myocardial intracellular edema induced by St. Thomas' Hospital cardioplegic solution. J Mol Cell Cardiol. 1991;23:1215-21.

12. O'Donnell ME. Role of Na-K-Cl cotransport in vascular endothelial cell volume regulation. Am J Physiol. 1993;264:C1316-26.

13. Masroor S, Lombardi P, Tehrani H, Yassin SF, Katariya K, Salerno TA. Beating-heart valve surgery in patients with renal failure requiring hemodialysis. J Heart Valve Dis. 2004;13:302-6.

14. Di Luozzo G, Panos AL, Lombardi P, Salerno TA. Simultaneous antegrade/retrograde normothermic perfusion with blood (beating heart) for aortic root replacement in acute type-a dissection of the aorta. J Card Surg. 2005;20:350-2.

15. Partington MT, Acar C, Buckberg GD, Julia P, Kofsky ER, Bugyi HI. Studies of retrograde cardioplegia. I. Capillary blood flow distribution to myocardium supplied by open and occluded arteries. $J$ Thorac Cardiovasc Surg. 1989;97:605-12.

16. Partington MT, Acar C, Buckberg GD, Julia PL. Studies of retrograde cardioplegia. II. Advantages of antegrade/retrograde cardioplegia to optimize distribution in jeopardized myocardium. $J$ Thorac Cardiovasc Surg. 1989;97:613-22.

17. Ihnken K, Morita K, Buckberg GD, Aharon A, Laks H, Panos AL, et al. The safety of simultaneous arterial and coronary sinus perfusion: experimental background and initial clinical results. J Card Surg. 1994;9:15-25.

18. Ihnken K, Morita K, Buckberg GD, Aharon A, Laks H, Beyersdorf F, et al. Simultaneous arterial and coronary sinus cardioplegic perfusion: an experimental and clinical study. Thorac Cardiovasc Surg. 1994;42: 141-7.

19. Barry JA, McGovern KA, Lien YH, Ashmore B, Gillies RJ. Dimethyl methylphosphonate (DMMP): $\mathrm{a}^{31} \mathrm{P}$ nuclear magnetic resonance spectroscopic probe of intracellular volume in mammalian cell cultures. Biochemistry. 1993;32:4665-70.

20. Fisher MJ, Dillon PF. Phenylphosphonate: a ${ }^{31}$ P-NMR indicator of extracellular $\mathrm{pH}$ and volume in the isolated perfused rabbit bladder. Circ Res. 1987;60:472-7.

21. Li G, Xiang B, Dai G, Shaw A, Liu H, Yang B, et al. Tissue edema does not change gadolinium-diethylenetriamine pentaacetic acid (GdDTPA)-enhanced T1 relaxation times of viable myocardium. J Magn Reson Imaging. 2005;21:744-51.

22. Mauney MC, Kron IL. The physiologic basis of warm cardioplegia. Ann Thorac Surg. 1995;60:819-23.

23. Salerno TA, Houck JP, Barrozo CA, Panos A, Christakis GT, Abel JG, et al. Retrograde continuous warm blood cardioplegia: a new concept in myocardial protection. Ann Thorac Surg. 1991;51:245-7.

24. Misare BD, Krukenkamp IB, Lazer ZP, Levitsky S. Recovery of postischemic contractile function is depressed by antegrade warm continuous blood cardioplegia. J Thorac Cardiovasc Surg. 1993;105: $37-44$.

25. Taira A, Yamashita M, Arikawa K, Hamada Y, Toyohira H, Akita H. Cardiac lymph in electrical ventricular fibrillation: an experimental study. Ann Thorac Surg. 1979;27:144-7.

26. Mehlhorn U, Allen SJ, Adams DL, Davis KL, Gogola GR, de Vivie ER, et al. Normothermic continuous antegrade blood cardioplegia does not prevent myocardial edema and cardiac dysfunction. Circulation. 1995;92:1940-6.

27. Laine GA, Granger HJ. Microvascular, interstitial, and lymphatic interactions in normal heart. Am J Physiol. 1985;249:H834-42.

28. Opie LH. Substrate and energy metabolism of the heart. In: Sperelakis $\mathrm{N}$, editor. Physiology and pathophysiology of the heart. 2nd ed. Boston: Kluwer Academic; 1989. p. 327-60

29. Stanley WC, Recchia FA, Lopaschuk GD. Myocardial substrate metabolism in the normal and failing heart. Physiol Rev. 2005;85:1093129.

30. Zhang J. Myocardial energetics in cardiac hypertrophy. Clin Exp Pharmacol Physiol. 2002;29:351-9. 